\title{
Characterization of the Mean Degree of Polymerization of Proanthocyanidins in Red Wines Using Liquid Chromatography-Mass Spectrometry (LC-MS)
}

\author{
S. González-Manzano, C. Santos-Buelga,* J. J. Pérez-Alonso, \\ J. C. Rivas-GonZalo, AND M. T. Escribano-BAILÓN \\ Grupo de Investigación en Polifenoles, Facultad de Farmacia, Unidad de Nutrición y Bromatología, \\ Universidad de Salamanca, E-37007 Salamanca, Spain
}

\begin{abstract}
An HPLC-MS method for the characterization of proanthocyanidins (PA) has been refined. Further application to red wines provided interesting conclusions about the composition of the flavanol fraction and PA extractability during winemaking. The yield in PA extraction increases with the length of the postfermentative maceration (PFM), as well as the mean degree of polymerization (mDP) of wine flavanols. In early winemaking events mostly monomers to trimers are extracted from grape solids, whereas PFM is required for the significant extraction of higher oligomers. Nevertheless, at the end of a regular process of elaboration the mDP is not very high and does not usually exceed a value of 2.3, dimers and trimers being the predominant flavanols in red wines. With regard to groups of compounds, gallocatechins and prodelphinidins (located in the skins) are extracted rapidly in the first stages of the winemaking. On the contrary, long postfermentative macerations are required for the extraction of galloyled derivatives from the seeds. PA extractability is also dependent on the grape variety used for winemaking. Thus, wines made with Graciano grapes were found to require a longer process of PFM than those made from Tempranillo grapes to obtain similar yields in the extraction of flavanols.
\end{abstract}

KEYWORDS: Proanthocyanidins; mean degree of polymerization; wine; LC-MS

\section{INTRODUCTION}

Proanthocyanidins (PAs) are polymeric flavan-3-ols having elementary units usually linked by $\mathrm{C}-\mathrm{C}$ and occasionally $\mathrm{C}-\mathrm{O}-\mathrm{C}$ bonds. They are widespread in plants, where they play a crucial role in the mechanisms of natural defense, giving them protection against pathogens and acting as predator repellents (1). They are also found in food of plant origin, in particular, in fruits, legume seeds, cereal grains, and different beverages (wine, tea, cocoa, cider), where they contribute to functional and sensory properties. The characteristic that best defines the PAs is their capacity to join to substances such as proteins, polysaccharides, alkaloids, free radicals, and metallic ions. Many of their biological activities derive from these properties. It is precisely their capacity to precipitate salivary proteins that confers on them an astringent character (2). In grapes, they are located fundamentally in seed, skin, and stem; from there they pass to the wine during winemaking. In wine, these compounds are directly responsible for sensory properties such as body, bitterness, and astringency (3-5) and contribute to others, such as color, through their interactions with the anthocyanins. The processes of copigmentation with the anthocyanins and the formation of new pigments, in which PAs are involved, are

* Author to whom correspondence should be addressed (telephone +34 923 294537; fax + 34923 294515; e-mail csb@usal.es). necessary phenomena for the stability and definition of red wine color $(6,7)$. All of these processes are still insufficiently known, and their study demands a wider and more detailed knowledge of the PA composition of the grape and wine. Furthermore, PAs are increasingly recognized as having beneficial health effects for humans (2). Recent studies suggest that only the low molecular weight oligomers (polymerization degree up to 3 ) might be absorbed in the gastrointestinal tract ( 8 ). Considering that it appears that the polymeric PAs are not depolymerized in the stomach (9), those foodstuffs that present PAs with a low degree of polymerization could prove to be especially interesting.

Most of the methods used to determine the degree of polymerization of PAs are based on depolymerization in the presence of nucleophiles (10-15), many of which present the inconvenience of being difficult or uncomfortable to work with and the added problem of the instability of the adducts formed $(16,17)$

The aim of this work was to optimize a methodology for the qualitative and quantitative characterization of wine proanthocyanidins using HPLC-MS. The method was applied to the study of the PA fraction during winemaking, so that conclusions about tannin extractability could be obtained. 


\section{MATERIALS AND METHODS}

Chemicals and Standards. All organic solvents utilized were of HPLC grade and were purchased from Merck, Darmstadt, Germany. Standards of catechin (C), epicatechin (EC), epicatechin-3-O-gallate (ECG), and malvin (i.e., malvidin-3,5-diglucoside) were purchased from Sigma, St. Louis, MO.

Samples. The wines were supplied by the cellar Roda S.A. located in Haro (La Rioja, Spain). They were obtained from Vitis vinifera red grapes, harvested in 2003, of the Tempranillo variety (wine 1) and from a blend of the Garnacha and Graciano red varieties in a proportion of 70:30 (wine 2). The winemaking process was carried out at industrial scale $(17000 \mathrm{~L})$ and was the same in both wines. Briefly, it includes cold prefermentative maceration $\left(14^{\circ} \mathrm{C}, 2\right.$ days $)$, alcoholic fermentation at controlled temperature $\left(23 \pm 2{ }^{\circ} \mathrm{C}\right)$, and postfermentative maceration at $20{ }^{\circ} \mathrm{C}$. Maceration was facilitated by daily pumping-over and pushing-down the pomace cap with oxygenation during alcoholic fermentation and without air in further stages. Afterward, the wines were transferred to French oak barrels $(225 \mathrm{~L})$ for the malolactic fermentation $\left(20{ }^{\circ} \mathrm{C}, 38\right.$ days on average) and aging at $9-12{ }^{\circ} \mathrm{C}$. Samples were collected at the end of the cold prefermentative maceration, after the alcoholic fermentation, at 3, 7, and 11 days of postfermentative maceration, and after 11 months in French oak barrels.

Isolation of Proanthocyanidins. Grape seeds were utilized to isolate proanthocyanidins. The seeds were manually separated, washed with distilled water, and freeze-dried. The dry seeds were ground to obtain a homogeneous powder, $75 \%$ methanol in water was added and mixed by stirring, and the mixture was further maintained for $15 \mathrm{~min}$ in an ultrasonic bath at room temperature. Afterward, it was centrifuged for $10 \mathrm{~min}$, the supernatant was collected, and the residue was submitted twice more to the same extraction procedure. The methanolic extracts were combined and concentrated in vacuo at $30{ }^{\circ} \mathrm{C}$ until the elimination of the methanol. The aqueous extract obtained was washed repeatedly with hexane to eliminate liposoluble substances and fractionated on a Sephadex LH-20 column $(500 \times 30 \mathrm{~mm})$ using ethanol as a solvent as described in Escribano-Bailon et al. (18). The procyanidin dimers B2 (EC-4,8-EC), B1 (EC-4,8-C), and B2-3-O-gallate (EC-4,8-EC-3-Ogallate) and the trimer EEC (EC-4,8-EC-4,8-C) were isolated from the Sephadex LH-20 fractions by semipreparative HPLC. The C4-C8 epicatechin trimer (procyanidin $\mathrm{C} 1$ ) and the $\mathrm{C} 4-\mathrm{C} 8$ epicatechin tetramer were isolated from apple peel using the same procedure.

Semipreparative HPLC. A Waters 600 series pump and a Phenomenex $5 \mu \mathrm{m}$ Ultracarb ODS20 $(10 \times 250 \mathrm{~mm})$ column were used. The solvents were (A) acetic acid 5\% and (B) methanol. The gradient used was $0-10 \%$ B over $10 \mathrm{~min}, 10-20 \%$ B over $20 \mathrm{~min}, 20-50 \%$ B over $20 \mathrm{~min}, 50-75 \%$ B over $5 \mathrm{~min}$, and $75-100 \%$ B over $5 \mathrm{~min}$. Detection was carried out at $280 \mathrm{~nm}$, and the peaks were collected by a fraction collector. The fractions that contained the PAs were concentrated under vacuum at low temperature and further lyophilized. The purity and identity of the isolated PA were checked by HPLCDAD and LC-MS by comparison with standards previously obtained in our laboratory (19).

Analysis of Flavanols. The analyses were carried out by HPLC using a Hewlett-Packard 1100 chromatograph with a quaternary pump and a diode array detector (DAD) coupled to an HP Chem Station (rev. A.05.04) data-processing station. A Waters Spherisorb S3 ODS-2 C8, $3 \mu \mathrm{m}(4.6 \times 150 \mathrm{~mm})$ column was used thermostated at $30{ }^{\circ} \mathrm{C}$. The mobile phase was (A) $2.5 \%$ acetic acid, (B) acetic acid/acetonitrile (10: $90, \mathrm{v}: \mathrm{v})$, and (C) acetonitrile. The elution gradient established was $100 \%$ A to $100 \%$ B over $5 \mathrm{~min}, 0-15 \% \mathrm{C}$ in B over $25 \mathrm{~min}$, and $15-50 \%$ $\mathrm{C}$ in $\mathrm{B}$ over $10 \mathrm{~min}$. MS detection was also performed using a Finnigan LCQ detector (San Jose, CA) equipped with an ESI source and an ion trap mass analyzer, which were controlled by the LCQ Xcalibur software. The mass spectrometer was connected to the HPLC system via the DAD cell outlet. Both the auxiliary and the sheath gases were nitrogen at flow rates of 6 and $1.2 \mathrm{~L} \mathrm{~min}^{-1}$, respectively. The source voltage was $4.5 \mathrm{kV}$, the capillary voltage was $28 \mathrm{~V}$, and the capillary temperature was $270{ }^{\circ} \mathrm{C}$. Spectra were recorded in positive ion mode between $\mathrm{m} / \mathrm{z}, 150$ and 2000. The MS detector was programmed to perform a series of three consecutive scans: a full mass scan, an MS/
MS scan of the most abundant ion the first scan, and an $\mathrm{MS}^{3}$ of the most abundant ion in the $\mathrm{MS}^{2}$ using normalized collision energy of $45 \%$.

Preparation of Wine samples for $\mathrm{mDP}$ Analysis. With the objective of eliminating the anthocyanins, $2 \mathrm{~mL}$ of wine diluted (1:1) with $0.1 \mathrm{~N} \mathrm{HCl}$ was placed in an Oasis MCX cartridge previously conditioned with $5 \mathrm{~mL}$ of methanol and $5 \mathrm{~mL}$ of water. After washing with water, the flavan-3-ols were eluted with $15 \mathrm{~mL}$ of methanol, the anthocyanins being retained in the cartridge. A small volume of water was added to the eluate and concentrated under vacuum at temperature lower than $30{ }^{\circ} \mathrm{C}$ until the complete elimination of the methanol. The volume of the aqueous residue was adjusted to $450 \mu \mathrm{L}$ with distilled water. A further $50 \mu \mathrm{L}$ of a solution of malvin (internal standard) was added to provide a final concentration of the anthocyanin of $0.025 \mathrm{mM}$.

Analysis of the PA Fraction in Wine. The average full mass spectrum of a given wine sample was obtained from the chromatogram recording between 0 and $40 \mathrm{~min}$, sufficient time for the flavanols to elute from the column in the HPLC conditions used. Calibration curves (flavanol concentration versus relative abundance) were obtained using flavan-3-ol monomers (C, EC, ECG), dimers (procyanidins B1, B2, and $\mathrm{B} 2-3-\mathrm{O}$-gallate), trimers (EEC and $\mathrm{C} 1$ ), and the $\mathrm{C} 4-\mathrm{C} 8$ epicatechin tetramer, in the range of concentration of $0.005-0.2 \mathrm{mM}$. In all cases, malvin at a concentration of $0.025 \mathrm{mM}$ was used as an internal standard. The quantification of flavanols of each group (i.e., monomers, galloylated monomers, dimers, galloylated dimers, trimers, and tetramers) in wines was made by comparing the intensities of their ion signals recorded in the full mass spectrum of the sample with the corresponding calibration curve.

The concentration of total flavanols in wine was calculated from the sum of the individual concentrations obtained for each of the different groups of flavanols analyzed, expressed in milligrams per liter.

The mean degree of polymerization $(\mathrm{mDP})$ of the flavanol fraction was estimated from the ratio between the total number ( $\mathrm{mmol}$ ) of elementary flavan-3-ol units for the whole of quantified flavanols and the amount (mmol) of total flavanols in the sample

$$
\mathrm{mDP}=\frac{\sum N_{\mathrm{i}} \times U_{\mathrm{i}}}{N_{\mathrm{t}}}
$$

where $N_{\mathrm{i}}$ is the amount (mmol) of flavanols of each group (i.e., monomers, galloylated monomers, dimers, galloylated dimers, trimers, and tetramers), $U_{\mathrm{i}}$ is the number of elementary units in each group of flavanols (i.e., 1, 2, 3, or 4 for monomers, dimers, trimers and tetramers, respectively), and $N_{\mathrm{t}}$ is the amount (mmol) of total flavanols in the sample $\left(=\Sigma N_{\mathrm{i}}\right)$.

Statistical Analysis. Analysis of the variance (ANOVA) was conducted using SPSS 9.0. Significant $(p<0.05)$ differences between means of three replicates were identified using Tukey's procedure.

\section{RESULTS AND DISCUSSION}

LC-MS Method for Proanthocyanidin Characterization. The PA composition in the wines was characterized from the average full mass spectra obtained after HPLC separation. Direct injection of the wine samples into the mass spectrometer was discounted because the ionization of the molecules is influenced not only by their nature and concentration but also by the nature and concentration of the rest of the compounds present simultaneously in the ionization chamber. After the chromatographic separation, each flavanol enters individually the ionization chamber, and thus the matrix effect is minimized. Proanthocyanidin detection can be made in either negative or positive ion mode; it is considered that better response is usually obtained in negative mode (20). Nevertheless, the presence of acetic acid as a chromatographic solvent improves the efficiency of the ionization in positive mode, whereas it may favor the formation of acetate/phenolate adducts in negative mode, which sometimes makes the interpretation of the spectra more difficult. Taking this into account, as well as the good results obtained 

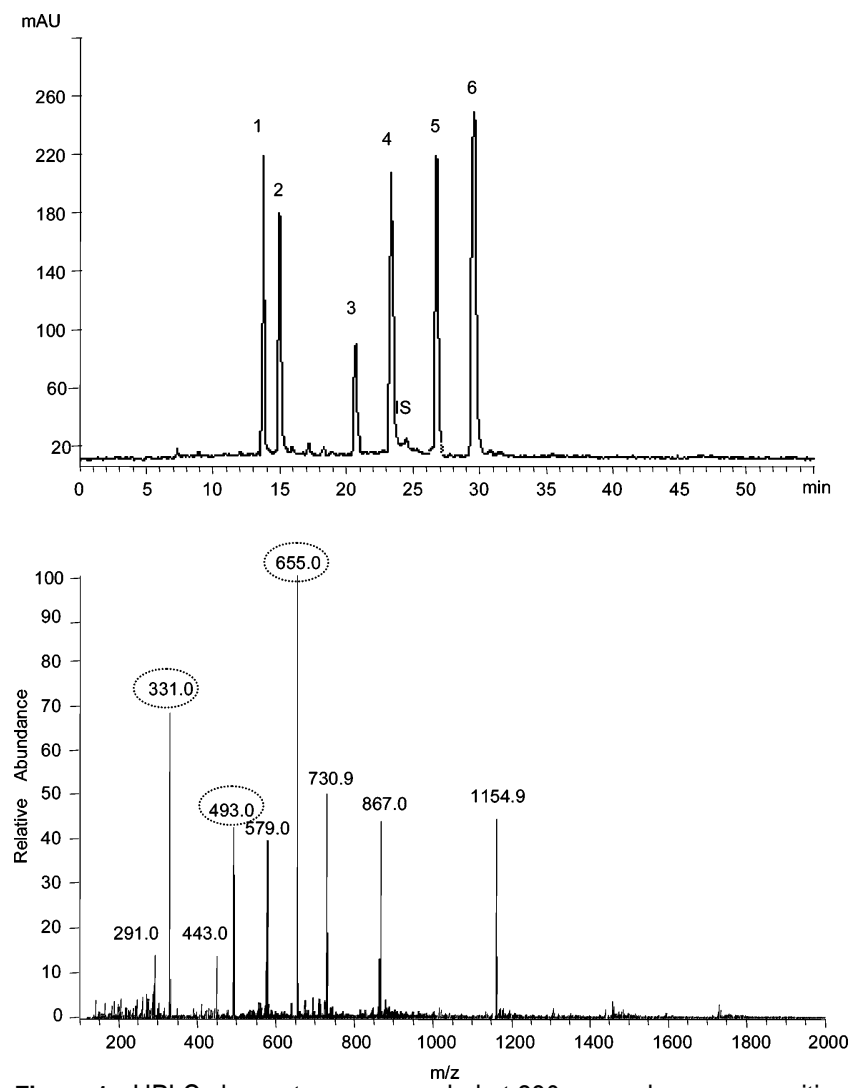

Figure 1. HPLC chromatogram recorded at $280 \mathrm{~nm}$ and average positive full mass spectrum obtained for a solution of flavan-3-ol standards (concentration of each compound $=0.1 \mathrm{mM}$ ). Peaks and positive molecular ions: (1) procyanidin B2 ( $\mathrm{m} / \mathrm{z} 579)$; (2) procyanidin C1 ( $\mathrm{m} / \mathrm{z}$ 867); (3) epicatechin ( $\mathrm{m} / \mathrm{z} 291)$; (4) epicatechin-3-O-gallate ( $\mathrm{m} / \mathrm{z} 443)$; (5) C4-C8 epicatechin tetramer ( $\mathrm{m} / \mathrm{z}$ 1155); (6) procyanidin B2-3-O-gallate $(\mathrm{m} / z 731)$. Ion signal at $\mathrm{m} / \mathrm{z} 655$ corresponds to malvin used as the internal standard (IS) and signals at $\mathrm{m} / \mathrm{z} 493$ and 331 (malvidin 3-glucoside and malvidin, respectively) derived from its fragmentation in the ion source.

in previous analysis of wine PAs (19), we decided to use the positive ion mode in this study.

Analyses were carried out using malvidin 3,5-diglucoside as internal standard (IS). The reason for choosing an anthocyanin is that they are charged molecules with an easy ionization compared to PAs. Therefore, at concentrations even smaller than those of the PAs, its peak is expected to be maximum in the mass spectrum, thus preventing saturation of the signals corresponding to flavan-3-ols and allowing them to be measured and related to their concentration. Malvin was selected due to its absence in Vitis vinifera red wines and commercial availability. In addition, it presents a time of chromatographic elution within the range in which the PAs elute in the HPLC conditions used. A malvin concentration of $0.025 \mathrm{mM}$ was employed, which provides a minimum peak compared to the ones of flavan3-ols in the HPLC chromatograms obtained with UV detection, whereas it always reaches $100 \%$ of relative abundance in the mass spectra even at the greatest flavan-3-ol concentrations assayed in this study.

Figure 1 shows the chromatogram recorded at $280 \mathrm{~nm}$ and its average full mass spectrum obtained for a standard solution of flavan-3-ols (concentration of each compound in the solution $0.1 \mathrm{mM})$ added with malvin $(0.025 \mathrm{mM})$ as IS. The ion signals observed in the mass spectrum correspond to EC $(\mathrm{m} / \mathrm{z}$ at 291$)$, ECG $(\mathrm{m} / \mathrm{z}$ at 443), procyanidins B2 ( $\mathrm{m} / \mathrm{z}$ at 579$), \mathrm{B} 2-3-O$-gallate $(\mathrm{m} / \mathrm{z}$ at 731$)$, and $\mathrm{C} 1(\mathrm{~m} / \mathrm{z}$ at 867$)$, and $\mathrm{EC}$ tetramer $(\mathrm{m} / \mathrm{z}$ at 1155$)$. The IS signal at $\mathrm{m} / \mathrm{z} 655$ corresponds to the IS (i.e., malvidin 3,5-diglucoside), and signals at $\mathrm{m} / \mathrm{z}, 493$ (malvidin 3-glucoside) and 331 (malvidin) are derived from its fragmentation in the ESI source.

Before the method was applied to flavanol characterization in wine samples, it was checked that no relevant fragmentation of the PA oligomers occurred in the ionization conditions used for mass detection. It was also checked that no signals that could be attributed to multiply-charged ions were produced, because the distance between the isotopic peaks of the carbon was always equal to 1 mass unit (21). Both aspects are important to ensure that PAs of different degrees of polymerization are adequately quantified. To check this, different PA standards were analyzed using the same LC-MS method further used for the wine samples. As an example, the chromatograms and mass spectra obtained for two procyanidin standards are shown in Figure 2.

For its use with quantitative purposes the method was calibrated with different flavan-3-ols: monomers (C, EC), dimers (procyanidins B1 and B2), trimers (EEC and C1), C4-
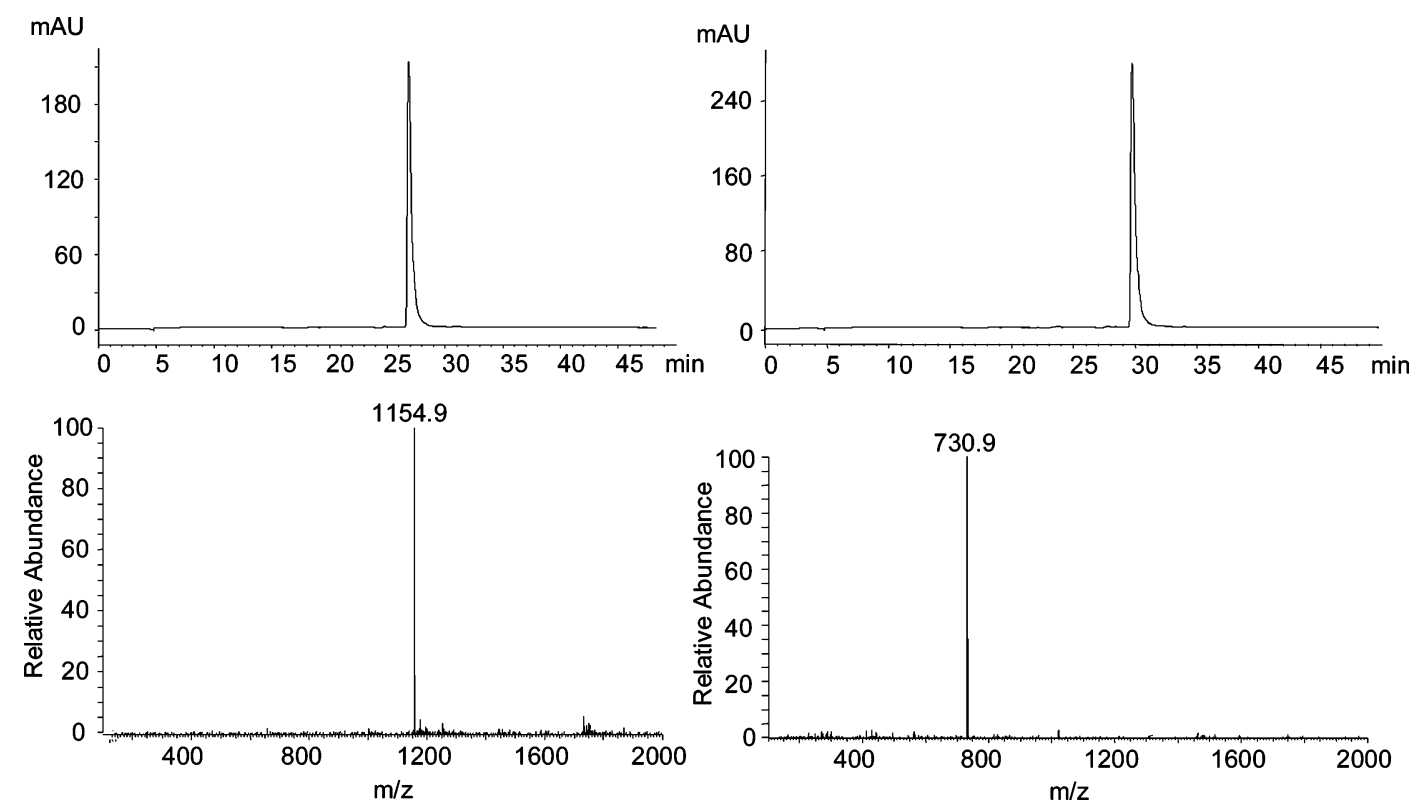

Figure 2. HPLC cromatograms recorded at $280 \mathrm{~nm}$ and full mass spectra obtained for two procyanidin standards: C4-C8 epicatechin tetramer and procyanidin B2-3-O-gallate (left and right, respectively). 
Table 1. Calibration Curves Obtained for Different Groups of Flavanols ( $y=$ Relative Abundance of the Compound Signal in the Mass Spectrum; $x=$ Concentration Expressed in Millimolar)

\begin{tabular}{llc}
\hline \multicolumn{1}{c}{ type of flavanol } & \multicolumn{1}{c}{ equation } & $R^{2}$ \\
\hline catechin monomers & $y=124.75 x+3.49$ & 0.9990 \\
galloyled monomers & $y=56.09 x+2.0992$ & 0.9993 \\
procyanidin dimers & $y=324.43 x+3.5125$ & 0.9991 \\
galloyled dimers & $y=413.33 x+3.105$ & 0.9994 \\
procyanidin trimers & $y=350.6 x+4.9867$ & 0.9997 \\
procyanidin tetramers & $y=518.76 x+0.8258$ & 0.9976 \\
\hline
\end{tabular}
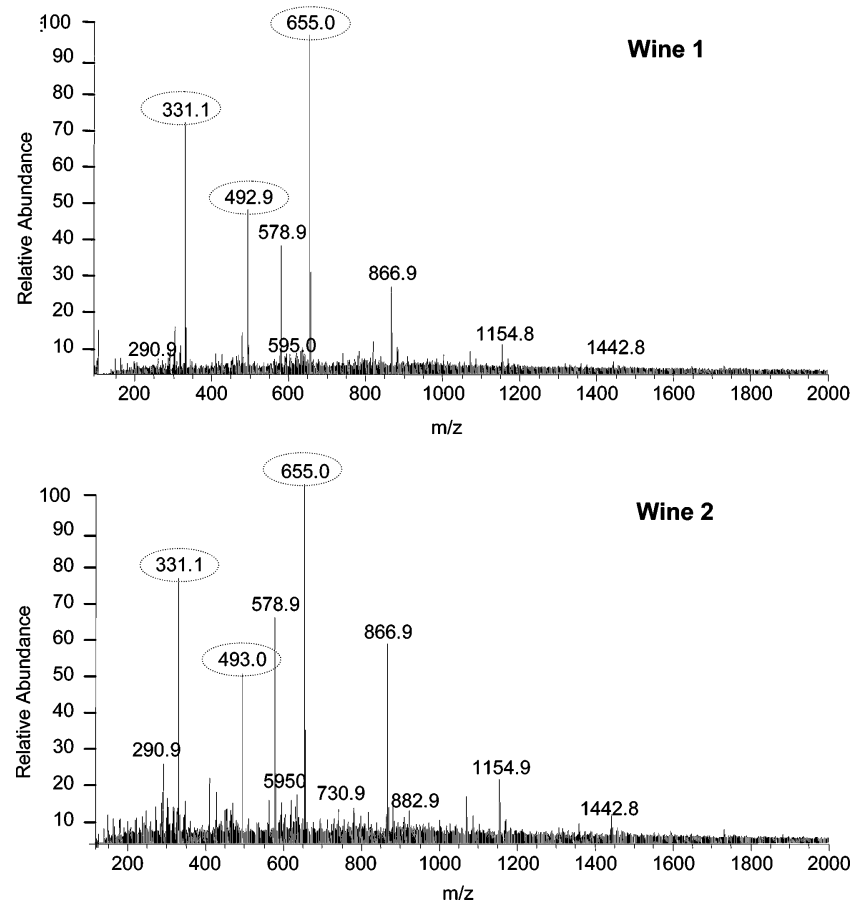

Figure 3. Average full mass spectra obtained for samples of wines 1 and 2 collected after 1 week of postfermentative maceration.

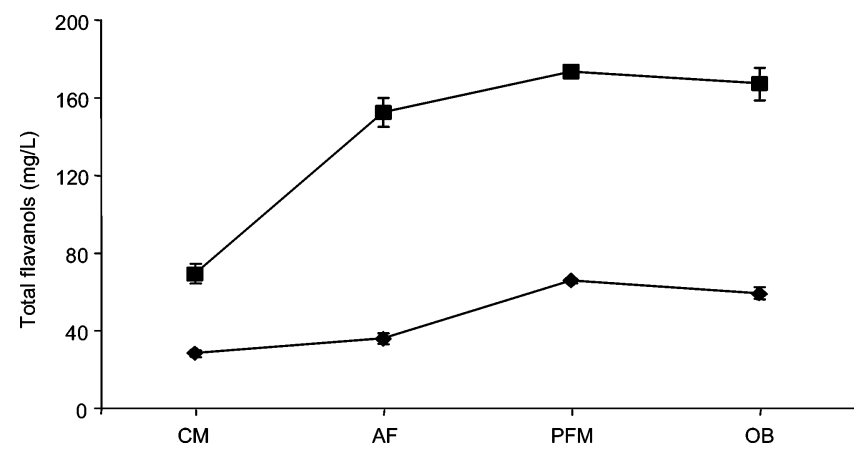

Figure 4. Change in the concentrations of total flavanols during winemaking in wines $1(\checkmark)$ and $2(\boldsymbol{\square})$. Samples were collected at the end of the prefermentative cold maceration (CM) after alcoholic fermentation (AF), after 1 week of postfermentative maceration (PFM), and after 11 months in oak barrels (OB).

C8 epicatechin tetramers, and galloyled derivatives (ECG and B2-3-O-gallate). The calibration curves obtained and further used for the quantification of the different flavanol groups in the wine samples are indicated in Table 1. Similar responses were obtained for the nongalloyled monomers ( $\mathrm{C}$ and $\mathrm{EC})$, dimers (B1 and B2), and trimers ( $\mathrm{C} 1$ and EEC). However, a different response was obtained for the distinct groups of flavanols. Thus, for the same molar concentration, the capacity of ionization was greater as the number of elementary flavanol
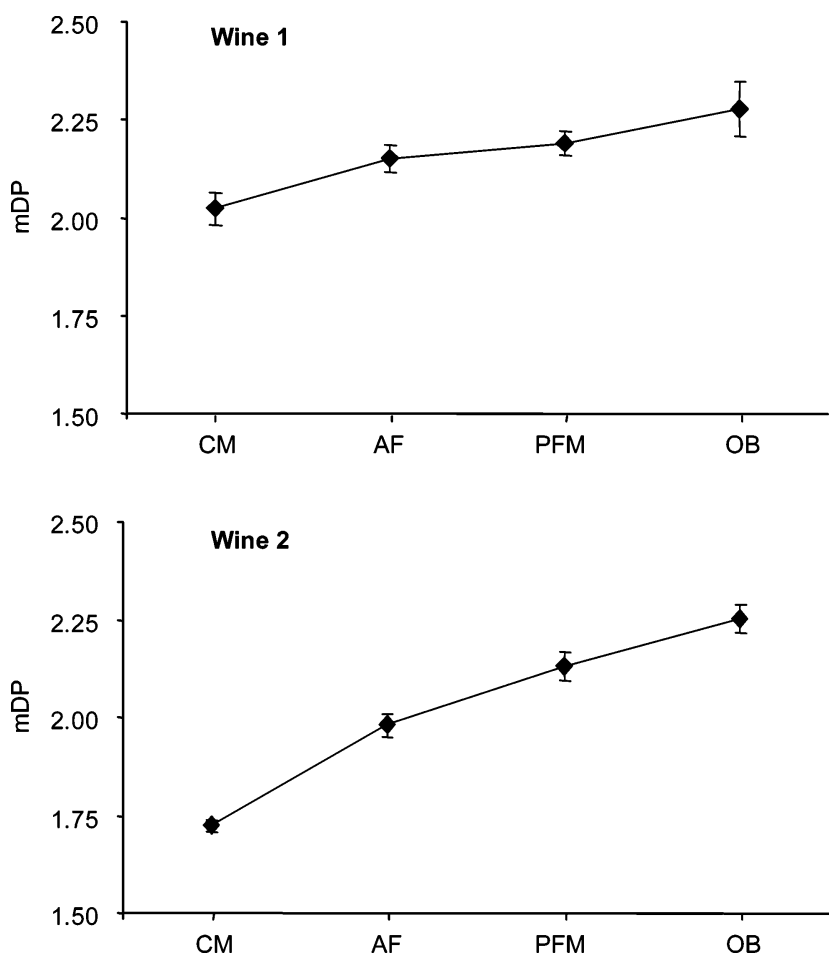

Figure 5. Change in the mean degree of polymerization (mPD) during winemaking in wines 1 (top) and 2 (bottom). Samples were collected at the end of the prefermentative cold maceration (CM) after alcoholic fermentation (AF), after 1 week of postfermentative maceration (PFM), and after 11 months in oak barrels (OB).

units in their structure increased. A similar observation was made by Cheynier et al. (21), using negative ion mode, who indicated that PAs are more easily charged as the degree of polymerization or the number of galloyl substituents increases. As for the galloyled flavanols here assayed, the capacity of ionization of the galloyled dimer was superior to that of the nongalloyled dimers, whereas the galloyled monomer (ECG) showed lower capacity of ionization than C and EC. No standards of gallocatechins and mixed PAs containing both diand trihydroxylated units in the $\mathrm{B}$ ring were available for calibration and, therefore, when present in the samples, they were quantified using the curves obtained for the equivalent catechins or procyanidin oligomers. Fulcrand et al. (22) indicated that the signal intensity within an ion peak series decreases as the number of trihydroxylated units increases. In such case, gallocatechins and mixed PAs would be underestimated in our samples.

Analysis of Wine Samples. Figure 3 shows the average full mass spectra corresponding to samples of wines 1 and 2 collected at the end of postfermentative maceration. Ion signals corresponding to catechin monomers and procyanidin dimers, trimers, tetramers, and pentamers could be observed in quantifiable levels, as well as some due to proanthocyanidins containing both di- and trihydroxylated units in the B ring. Thus, the signal at $m / z, 595$ is attributed to a dimer formed by a unit of catechin and another one of gallocatechin (19). The molecular ion at $\mathrm{m} / \mathrm{z} 883$ might fit either a trimer containing two catechin units and one gallocatechin unit or a digalloyled procyanidin dimer. However, the fact that hardly galloyled catechins or monogalloyled procyanidin dimers were detected in these particular wine samples led us to think that it corresponds to the mixed PA trimer. The absence of peaks corresponding to galloyled flavanols and the low levels of prodelphinidins detected in these 

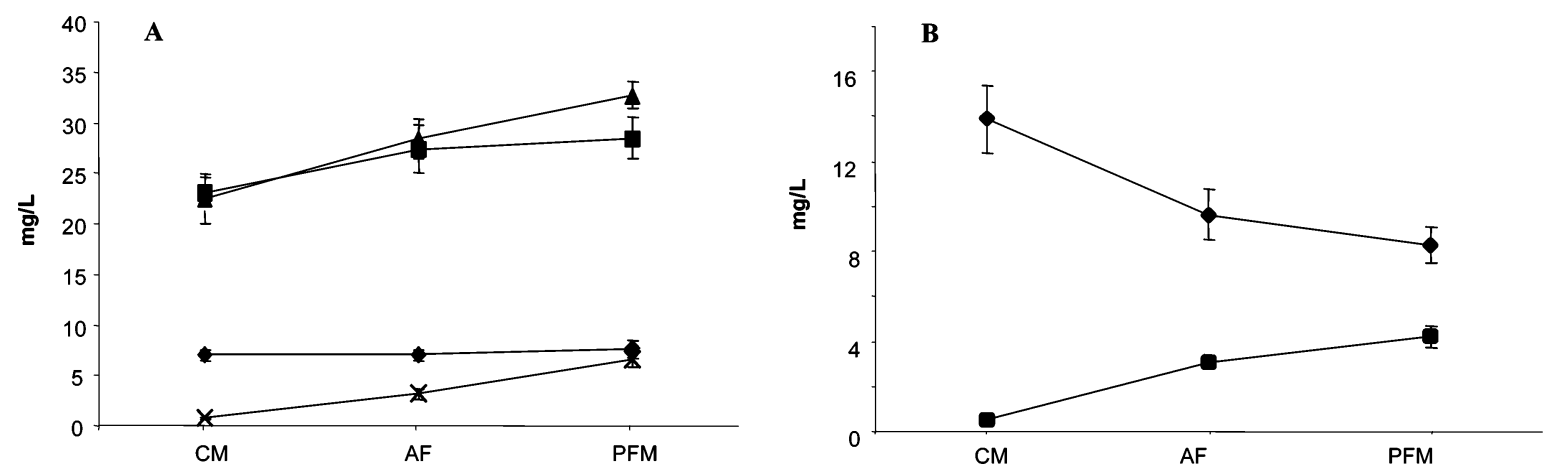

Figure 6. Change in the concentrations of $(\mathbf{A})$ flavanol monomers $(\bullet)$ and procyanidin dimers $(\boldsymbol{\square})$, trimers $(\mathbf{A})$, and tetramers $(X)$ and $(\mathbf{B})$ prodelphinidins $(\checkmark)$ and galloyled derivatives $(\boldsymbol{\square})$ in wine 1 during winemaking. Samples were collected at the end of the prefermentative cold maceration (CM) after alcoholic fermentation (AF) and after 1 week of postfermentative maceration (PFM).

wines are in agreement with previous findings in assays made in model solutions (23).

As has been previously indicated, PAs should be more easily charged as the degree of polymerization increases and signals corresponding to multiply-charged ions may appear. This means that if large oligomers are present in the wines in significant amounts, they should be observed in the mass spectra. In our case, the hexamers were the highest PAs detected in the wine samples, although always in very low nonquantifiable levels. In all cases, the signals of the different oligomers corresponded to monocharged ions, and their intensity in the full MS spectra progressively decreased for compounds above the trimer. No relevant signals that could be attributed to multiply-charged ions of oligo/polymers were observed in the samples analyzed. All in all, we can conclude that the detected oligomers are the main PAs and that no larger compounds are present in the wines analyzed at least in relevant amounts. Therefore, no essential information concerning wine tannin composition is missing by using the applied methodology.

To evaluate the behavior of the flavanol fraction during winemaking, samples of wines 1 and 2 collected at different vinification stages were analyzed. Figure 4 shows the changes in the concentration of total flavanols in those wines. Logically, the concentration of flavanols increases while pomace contact exists, with maximum levels situated at the end of the postfermentative maceration. In wine $2(160 \pm 2 \mathrm{mg} / \mathrm{L})$ a much greater content of flavanols was reached than in wine $1(65 \pm 4 \mathrm{mg}$ / L). This is in agreement with the greater concentration of flavanols existing in Graciano grapes and its higher percentage of solids (i.e., skin and seeds) in relation to pulp compared with Tempranillo's $(24,25)$. The changes produced in PA composition during winemaking were not only quantitative but also qualitative. Thus, an increase in the $\mathrm{mDP}$ of the flavanol fraction is produced during winemaking (Figure 5), which continues during oak storage. This increase of the polymerization during wine maturation, once the maceration with grape solids is over, is attributed to rearrangements in PA structure and their interaction with anthocyanins $(6,7)$.

Monomers to trimers were already present in the wines at the end of the cold prefermentative maceration. However, whereas no significant increase was produced in the levels of monomers in further winemaking stages, a progressive extraction of oligomers occurs, which is particularly important in the case of the trimers and tetramers (Figure 6A). It was also observed that the extraction of galloyled derivatives is produced very slowly. These compounds are less soluble than the corresponding nongalloyled derivatives, and they are mostly located in the seed, thus requiring longer macerations for significant extraction (23). On the contrary, the extraction of the gallocatechins and

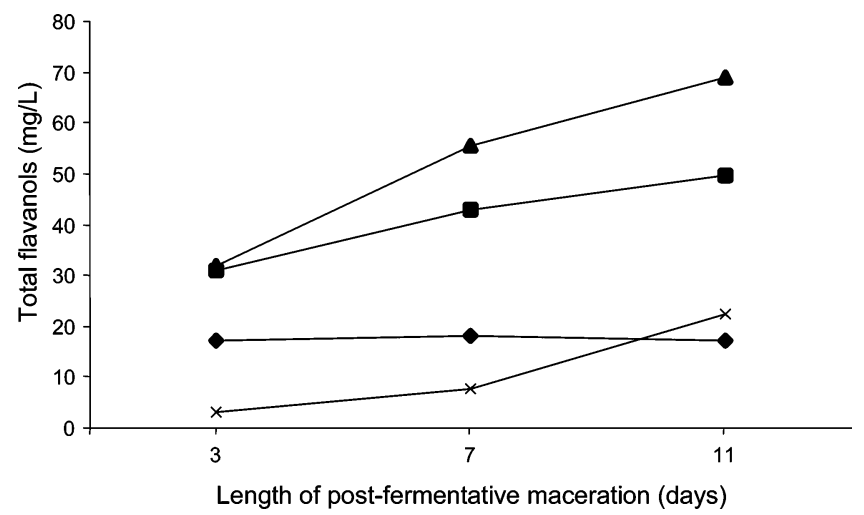

Figure 7. Change in the concentrations of flavanol monomers $(\bullet)$ and procyanidin dimers $(\boldsymbol{\square})$, trimers $(\mathbf{\Delta})$, and tetramers $(x)$ in wine 2 during postfermentative maceration.

prodelphinidins, located only in the skin, takes place quickly in the first stages of winemaking to further decline (Figure 6B).

The influence of the postfermentative maceration (PFM) in PA extraction was also assessed using three different PFM lengths: 3, 7, and 11 days. The longer the PFM, the greater was the extraction of oligomers, whereas no increase was produced in monomers (Figure 7). All of these observations are in agreement with our previous findings in model maceration assays (23).

It is noticeable that the size of the PAs found in the studied wines was not very high. Trimers and dimers were the predominating compounds, whereas no significant amounts of PAs greater than pentamers were observed. Thus, the mDP of the flavanol fractions in these wines did not exceed values of 2.3. These values are lower than those found by other authors using methods based on PA depolymerization (e.g., thiolysis). Thus, mDPs of 3.1-3.4 and 5-6 were found in French wines by Souquet et al. (26) and Sarni-Manchado et al. (27), respectively, and $\mathrm{mDP}$ values in the range of 6-13 were determined in Spanish and Portuguese wines $(25,28)$. However, in our experience, the results obtained using thiolysis may not be totally accurate, due to variable yields in PA cleavage and the formation of thioether adducts; side-products are also produced. In addition, for the quantification of the products released after thiolysis, previous calibration is required with the thioether adducts of the different (gallo)catechin units, which is not always easily achieved because they have to be previously synthesized and purified.

It is known that flavanols can act as anthocyanin copigments (29-31). In agreement with Boulton (32) this characteristic could have a twofold role. First, the binding of free anthocyanins 

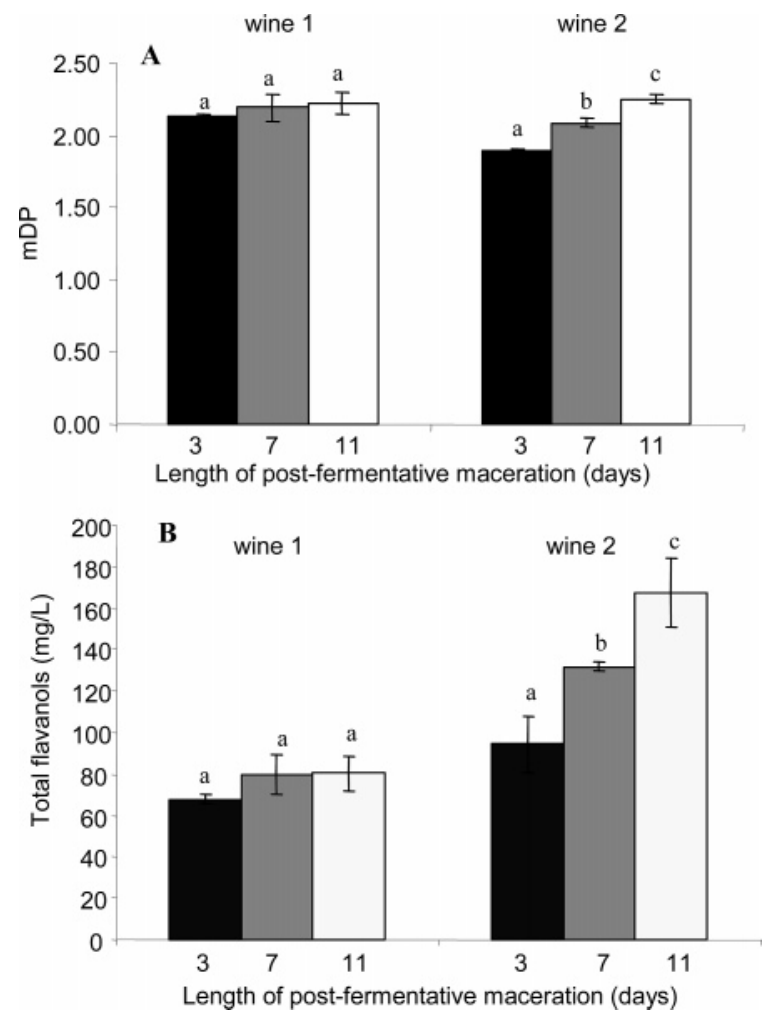

Figure 8. Change in (A) mean degree of polymerization and (B) concentration of total flavanols during postfermentative maceration (PFM) in wines 1 and 2. Different letters indicate significant differences $(p<$ 0.05).

into copigmented forms would enable more pigment to be retained in wine, and, second, the copigmented anthocyanins provide much more color than they would have in the free form. From this point of view, a greater extraction of flavanols should hold interest not only regarding wine astringency but also for anthocyanin retention and color. In this respect, a longer PFM, as long as it provides an increased extraction of flavanols, is expected to improve wine color definition and stability. However, it is necessary to take into account the fact that the extraction of PAs is not only dependent on the length of the maceration but also influenced by the characteristics of the grapes and the winemaking process. In Figure $\mathbf{8}$ it can be observed that in wine 1 no significant increase in PA concentration and $\mathrm{mDP}$ takes place after a week of PFM; however, PA levels and $\mathrm{mDP}$ continue increasing in wine 2 for at least 11 days. Because a similar winemaking process was used in both wines, this should be explained by the type of grapes used in each case. In this respect, the grapes of Graciano (wine 2), with a smaller size and greater proportion of seeds in relation to the must, would benefit from a longer PFM than Tempranillo's (wine 1), to obtain better yields in the extraction of flavanols. For that reason, the optimum length of the PFM must be determined for each particular case, depending on the type of grapes and the winemaking conditions.

\section{ACKNOWLEDGMENT}

We thank G. H. Jenkins for assistance with the English version of the manuscript.

\section{LITERATURE CITED}

(1) Haslam, E. Plant Polyphenols-Vegetable Tannins Revisited; Cambridge University Press: Cambridge, U.K., 1989; 230 pp.
(2) Santos-Buelga, C.; Scalbert, A. Proanthocyanidins and tanninlike compounds-nature, occurrence, dietary intake and effects on nutrition and health. J. Sci. Food Agric. 2000, 80, 10941117.

(3) Arnold, R. A.; Noble, A. C.; Singleton, V. L. Bitterness and astringency of phenolic fractions in wine. J. Agric. Food Chem. 1980, 28, 675-678.

(4) Noble, A. C. Bitterness and astringency in wine. In Bitterness in Foods and Beverages; Rouseff, R., Ed.; Elsevier: Amsterdam, The Netherlands, 1990; pp 145-158.

(5) Vidal, S.; Francis, L.; Noble, A.; Kwiatkowski, M.; Cheynier, V.; Waters, E. Taste and mouth-feel properties of different types of tannin-like polyphenolic compounds and anthocyanins in wine. Anal. Chim. Acta 2004, 513, 57-65.

(6) Haslam, H. In vino veritas: oligomeric procyanidins and the aging of red wines. Phytochemistry 1980, 19, 2577-2582.

(7) Somers, T. C. The polymeric nature of wine pigments. Phytochemistry 1971, 10, 2171-2182.

(8) Manach, C.; Williamson, G.; Morand, C.; Scalbert, A.; Rémésy, C. Bioavailability and bioefficacy of polyphenols in humans. I. Review of 97 bioavailability studies. Am. J. Clin. Nutr. 2005, 81, 230S-242S.

(9) Rios, L. Y.; Bennett, R. N.; Lazarus, S. A.; Remesy, C.; Scalbert, A.; Williamson, G. Cocoa procyanidins are stable during gastric transit in humans. Am. J. Clin. Nutr. 2002, 76, 1106-1110.

(10) Geissman, T. A.; Yoshimura, N. N. Synthetic proanthocyanidins. Tetrahedron Lett. 1996, 24, 2669-2673.

(11) Guyot, S.; Marnet, N.; Laraba, D.; Sanoner, P.; Drilleau, J.-F. Reversed-phase HPLC following thiolysis for quantitative estimation and characterization of the four main classes of phenolic compounds in different tissue zones of a French cider apple variety (Malus domestica Var. Kermerrien). J. Agric. Food Chem. 1998, 46, 1698-1705.

(12) Matthews, S.; Mila, I.; Scalbert, A.; Pollet, B.; Lapierre, C.; Herve du Penhoat, C. L. M.; Rolando, C.; Donnelly, D. M. X. Methods for estimation of procyanidins based on their acid polimerization in the present of nucleophiles. J. Agric. Food Chem. 1997, 45, 1195-1201.

(13) Prieur, C.; Rigaud, J.; Cheynier, V. Oligomeric and polymeric procyanidins from grape seeds. Phytochemistry 1994, 36, 781784.

(14) Thompson, R. S.; Jacques, D.; Haslam, E.; Tanner, R. I. N. Plant procyanidin. Part I. Introduction: isolation, structure and distribution in nature of plant procyanidins. J. Chem. Soc., Perkin Trans. 1 1972, 1387-1398.

(15) Torres, J. L.; Lozano, C. Chromatographic characterization of proanthocyanidins after thiolysis with cysteamine. J. Chromatogr. A 2001, 54, 523-526.

(16) Hemingway, R. W. Reaction at the interflavanoid bond of proanthocyanidins. In Chemistry and Significance of Condensed Tannins; Hemingway, R. W., Karchesy, J. J., Eds.; Plenum Press: New York, 1989; pp 345-368.

(17) Kennedy, J. A.; Jones, G. P. Analysis of proanthocyanidin cleavage products following acid-catalysis in the presence of excess phloroglucinol. J. Agric. Food Chem. 2001, 49, 17401746.

(18) Escribano-Bailon, T.; Gutierrez-Fernandez, Y.; Rivas-Gonzalo, J. R.; Santos-Buelga, C. Characterization of procyanidins of Vitis vinifera variety Tinta del Pais grape seeds. J. Agric. Food Chem. 1992, 40, 1794-1799.

(19) de Pascual-Teresa, S.; Rivas-Gonzalo, J. C.; Santos-Buelga, C. Prodelphinidins and related flavanols in wine. Int. J. Food Sci. Technol. 2000, 35, 33-40.

(20) Fulcrand, H.; Doco, T.; EsSafi, N. E. Study of the acetaldehyde induced polymerisation of flavan-3-ols by liquid chromatography ion spray mass spectrometry. J. Chromatogr. A 1996, 752, 8591

(21) Cheynier, V.; Doco, T.; Fulcrand, H.; Guyot, S.; Le Roux, E.; Souquet, J. M.; Rigaud, J.; Moutounet, M. ESI-MS analysis of polyphenolic oligomers and polymers. Analusis 1997, 25, M32M37. 
(22) Fulcrand, H.; Remy, S.; Souquet, J. M.; Cheynier, V.; Moutounet, M. Study of wine tannin oligomers by on-line liquid chromatography electrospray ionization mass spectrometry. J. Agric. Food Chem. 1999, 47, 1023-1028.

(23) Gonzalez-Manzano, S.; Rivas-Gonzalo, J. C.; Santos-Buelga, C. Extraction of flavan-3-ols from grape seed and skin into wine using simulated maceration. Anal. Chim. Acta 2004, 513, 283289.

(24) Gonzalez-Manzano, S.; de Marino-Caba; de Pascual-Teresa, S.; Tomas, E.; Escribano-Bailon, T.; Santos-Buelga, C. Vinificación de las uvas Graciano y Tempranillo. Estudio de cuatro cosechas. In Avances en Ciencias y Técnicas Enológicas (Actas de las VIII Jornadas de los Grupos de Investigación Enológica-GIENOL '05); Alamo, M., Nevares, I., Eds.; ITACyL: Palencia, Spain, 2005; pp 327-329.

(25) Monagas, M.; Gomez-Cordoves, C.; Bartolome, B. Monomeric, oligomeric, and polymeric flavan-3-ol composition of wines and grapes from Vitis vinifera L. cv. Graciano, Tempranillo, and Cabernet-Sauvignon. J. Agric. Food Chem. 2003, 51, 64756481.

(26) Souquet, J. M.; Mazauric, J.; Meudec, E.; Preys, S.; Morel-Salmi, C.; Cheynier, V. Comparison of different methods of depolymerization to the characterization and quantification of proanthocyanidins in grape seed, grape skin and wine. In Polyphenols Communications (Proceedings of the XXII International Conference on Polyphenols-ICP 2004); Hoikkala, A., Soidinsalo, O., Wähälä, K., Eds.; Groupe Polyphenols: Helsinki, Finland, 2004; pp 693-694.
(27) Sarni-Manchado, P.; Deleris, A.; Avallone, S. Analysis and characterization of wine condensed tannins precipitated by proteins used as fining agent in enology. Am. J. Enol. Vitic. 1999, 50, 81-86.

(28) Sun, B. H.; Spranger, I.; Roque-do-Vale, F. Effect of different winemaking technologies on phenolic composition in Tinta Miuda red wines. J. Agric. Food Chem. 2001, 49, 5809-5816.

(29) Berke, B.; Freitas, V. Influence of procyanidin structure on their ability to complex with oenin. Food Chem. 2005, 90, 453-460.

(30) Brouillard, R.; Wigand, M. C.; Dangles, O.; Cheminat, A. pH and solvent effects on the copigmentation reaction of malvin with polyphenols, purine and pyrimidine derivatives. J. Chem. Soc., Perkin Trans. 2 1991, 1235-1241.

(31) Mirabel, M.; Saucier, C.; Guerra, C.; Glories, Y. Copigmentation in model wine solutions: occurrence and relation to wine aging. Am. J. Enol. Vitic. 1999, 50, 211-218.

(32) Boulton, R. The copigmentation of anthocyanins and its role in the color of red wine: a critical review. Am. J. Enol. Vitic. 2001, $52,67-87$.

Received for review February 16, 2006. Revised manuscript received April 13, 2006. Accepted April 17, 2006. Financial support received from CICYT (Grant ref. AGL2002-00167) and INIA (Grant ref. VIN03043-C3) is gratefully acknowledged.

JF060467E 